\title{
INDUSTRIE ET ENVIRONNEMENT Les préoccupations environnementales de l'industrie des corps gras
}

\author{
Industry and Environment Environmental concerns of oils and fats industry
}

Oléagineux, Corps Gras, Lipides. Volume 9, Numéro 5, 308-15, Septembre - Octobre 2002, La filière

Auteur(s) : Xavier PAGES, Fabrice BOSQUE, Institut des corps gras, Iterg, rue Monge, 33600 Pessac, France.

Author(s) : Xavier PAGES, Fabrice BOSQUE

Résumé : Les préoccupations environnementales de l'industrie des corps gras sont principalement rythmées par le renforcement des contraintes réglementaires et concernent tous les aspects environnementaux : effluents gazeux et aqueux, déchets, management, énergie. Ces aspects sont inégalement présents dans les différents sous-secteurs de l'industrie des corps gras; quelques exemples sont présentés. La réduction des émissions de COV (composés organiques volatils) et les nuisances olfactives sont une problématique concernant principalement l'activité de trituration, la lipochimie et la fonte de corps gras animaux. Des valeurs limites de consommation de solvant sont maintenant imposées réglementairement en extraction ; la prise en compte de problèmes de nuisance olfactive donnent lieu à l'amélioration des procédés de traitement d'air (corps gras animaux) ou à leur mise en place progressive (trituration de colza). La gestion des déchets reste une préoccupation essentielle pour l'ensemble de la profession et tout particulièrement pour les secteurs des corps gras animaux et la lipochimie, en raison de la remise en cause des filières d'élimination due au renforcement des contraintes réglementaires, mais aussi en raison des coûts de traitement. $\mathrm{Ce}$ poste ne doit pas être négligé par les entreprises : réduction à la source, recherche de filières pérennes, valorisation énergétique ou matière sont des éléments permettant également une réduction significative des coûts. La maîtrise de la consommation d'énergie est un élément important, voir stratégique, notamment dans le secteur de la trituration des graines oléagineuses. Des actions de sensibilisation permettent de poursuivre également l'effort auprès des PME, actuellement moins impliquées. En matière de traitement d'eau, des avancées notables sont observées depuis une décennie ; néanmoins certaines difficultés persistent, notamment dans les domaines des corps gras animaux et de la lipochimie.

Summary : Nowadays strict environmental regulations concern issues like waste water, gaseous and odorous emissions and different other waste products from oil and fats industry. Oils and fats production plants as well as lipochemistry and animal fats rendering, are concerned by VOC (volatile organic compounds) reduction. These plants may emit solvents and odorous substances which are to be reduced; new solvent consumption limits are set by EU regulation. Waste products are produced mainly from lipochemistry and animal rendering. Other specific products such as used bleaching clays are also generated during oils and fats refining. Environmental management is an important tendency which leads to a better understanding in order to solve different environmental issues. 
Mots-clés : environnement, effluents, déchets, trituration, raffinage, lipochimie, corps gras animaux.

Keywords : environment, lipochemistry, animal fats rendering, oils and fats production.

\section{ARTICLE}

La prise en compte des aspects environnementaux dans le domaine industriel n'est pas récente mais tend à s'accentuer sous la pression conjointe du législateur et de l'opinion publique. Aucune entreprise ne peut désormais se permettre de négliger cette dimension dans sa stratégie à long terme, mais surtout dans sa gestion quotidienne, sans prendre le risque d'en subir des conséquences financières importantes.

Dans l'industrie des corps gras, l'évolution environnementale est principalement rythmée par le renforcement des contraintes réglementaires, issues principalement de la transcription des directives communautaires, concernant tous les aspects : effluents gazeux et aqueux, déchets, management, énergie.

Ces préoccupations environnementales sont inégalement présentes dans les différents secteurs de l'industrie des corps gras comme nous le verrons à travers ce panorama réalisé à partir des études menées par l'Iterg. Nous aborderons tour à tour les problématiques liées à l'air, à travers la réduction des émissions de COV (composés organiques volatils) et les nuisances olfactives, la gestion des déchets liés aux process, l'aspect énergétique et enfin le cas plus spécifique de l'utilisation des fluides frigorigènes.

\section{La réduction des émissions de COV : I'utilisation de l'hexane comme solvant d'extraction}

Depuis le développement industriel de la technique d'extraction au sulfure de carbone d'huile d'olive à partir de grignons, mis au point par Deiss à Marseille en 1855 [1], de nombreux solvants d'extraction ont pu être utilisés en trituration de graines oléagineuses et dans la production de corps gras animaux. La situation actuelle est beaucoup plus fermée : de nombreux solvants ne sont plus utilisables pour des raisons de sécurité industrielle ou de toxicité.

Sous l'angle de la sécurité alimentaire, l'utilisation industrielle de solvants est réglementée par la directive européenne $n^{\circ}$ 88/344 du 13 juin 1988, portant sur la " fabrication des denrées alimentaires et leurs ingrédients ", transcrite dans le droit français par l'arrêté du 19 novembre 1990, dont l'arrêté du 22 juin 1998 constitue la dernière modification à ce jour. Dans ce cadre, l'emploi de I'hexane est possible en extraction et en fractionnement des corps gras.

Sous l'angle environnemental se sont rajoutées plus récemment des dispositions issues de la directive $n^{\circ} 99 / 13$, dite directive COV, relative à la réduction des émissions de composés organiques, transcrite dans le droit français par l'arrêté du 29 mai 2000. Ces dispositions imposent notamment à l'industriel une surveillance des émissions, la mise en place d'un plan de gestion de solvants et définissent des valeurs limites d'émission. Pour les corps gras, des valeurs spécifiques de consommation sont instaurées par tonne de graine triturée ou produit traité. Les valeurs à respecter en extraction et raffinage sont reportées dans le tableau 1. 
Rappelons, à titre de comparaison que les valeurs de consommation en 1967 étaient de 10 litres par tonne de graine traitée [2].

L'impact industriel et économique de ces dispositions n'est pas anodin : les installations de trituration récentes et de grande capacité respectent facilement ces valeurs; en revanche, pour les quelques unités de plus faible capacité, des investissements importants sont à prévoir et peuvent remettre en question la viabilité économique de cette activé.

En fin et en marge de notre propos, mais à intégrer à la réflexion actuellement menée sur l'avenir de la technologie d'extraction par solvant $[3,4]$, citons le récent classement du n-hexane en R62 (classement européenne risk and safety) : substance toxique pour la reproduction de catégorie 3 (catégorie des substances préoccupantes pour la fertilité dans l'espèce humaine et des substances préoccupantes pour l'homme en raison d'effets toxiques possibles sur le développement). Ce nouveau code vient s'ajouter à ceux dont est déjà affublé le n-hexane, ce qui ne peut à l'avenir qu'accentuer les contraintes liées à l'utilisation de ce solvant.

\section{Les nuisances olfactives}

Deux types d'activités génèrent principalement des nuisances olfactives : la production d'huile de colza par trituration, mais surtout la fonte de corps gras animaux.

L'impact de ces nuisances sur le voisinage est un paramètre pris en compte dans le choix de l'implantation des sites récents. Cependant, les sites plus anciens sont le plus souvent implantés en zone urbaine et peuvent provoquer une réelle gêne pour le voisinage.

\section{La production des huiles végétales}

Lors du procédé d'obtention de l'huile de colza à partir des graines, différentes étapes sont susceptibles de générer des odeurs : cuisson et pression des graines, séchage et refroidissement des tourteaux. De plus, lors de l'étape d'extraction à l'hexane de l'huile à partir des tourteaux, les effluents gazeux provenant de la désolvantisation et de la distillation sont, après condensation, introduits dans une colonne de lavage d'huile minérale destinée à diminuer leur teneur en hexane (étape d'absorption). On constate une émission d'odeur en sortie de cette colonne de lavage.

La trituration du colza est caractérisée par la présence de nitriles et d'isothiocyanates dans les effluents gazeux. Les effluents de cuisson et pression de la graine de colza contiennent des faibles teneurs de mercaptans, de dioxyde de soufre et des traces d'hydrogène sulfuré. L'acétaldéhyde est présent en quantités non négligeables dans les incondensables provenant du système d'absorption du process, qui sont également chargés en hydrogène sulfuré, méthyl-mercaptans et dioxyde de soufre [5].

Bien que les composés chimiques identifiés au niveau des différents effluents soient les mêmes, on constate une variation de la composition chimique, non seulement en fonction de l'étape du process, mais également suivant le site de production. 
En termes d'émission d'odeur, on observe une prédominance de l'effluent provenant de l'absorption. Les différentes étapes du process ont pu être classées par ordre croissant de débit d'émission : absorption, refroidissement des tourteaux, cuisson des graines, pression des graines.

Les producteurs d'huiles végétales ne sont généralement pas équipés de traitement d'odeurs. Seul un site en France, ayant une capacité de production importante, dispose d'un système spécifique de traitement des odeurs (incinération et biofiltration), ce qui permet une réduction notable du débit d'odeur. Un suivi des nuisances, avec la participation active de la population, a été mis en place.

Les pertes en hexane, traceur indirect de certaines nuisances olfactives, peuvent être limitées par l'utilisation d'équipements récents (désolvanteur du type Schumacher).

L'activité spécifique du raffinage des graines oléagineuses, quand celle-ci est exercée en milieu urbain, peut parfois générer une nuisance pour le voisinage. Un diagnostic peut alors s'avérer nécessaire pour identifier les sources d'émission olfactive. Les nuisances éventuellement générées à l'étape de désodorisation par les tours de refroidissement peuvent être réduites par la mise en place d'une réfrigération indirecte. L'activité de cassage des pâtes de neutralisation peut également être une source d'odeurs irritantes. En ce qui concerne les eaux résiduaires issues du raffinage, il est nécessaire de procéder à leur traitement rapide, afin d'éviter une dégradation trop importante lors du stockage. Les bacs peuvent être couverts afin d'éviter une dispersion des odeurs.

\section{La production des corps gras animaux}

Dans le domaine de la production de corps gras animaux, la nuisance est principalement due à la spécificité de la matière première d'origine organique utilisée, subissant une dégradation biologique et pouvant être altérée avant la réception, et à la spécificité du process utilisé (traitement thermique, notamment le séchage, produisant un gaz chaud et humide). Les sources d'émission identifiées sont : la réception des matières premières (tissus adipeux), le lavage des camions de réception des matières, la fonte de ces matières premières, I'unité de traitement des effluents aqueux, I'unité de traitement des effluents gazeux (quand celle-ci subit des dysfonctionnements) et éventuellement le raffinage de la graisse [6].

L'effluent est très majoritairement composé d'ammoniac, puis d'hydrogène sulfuré, d'acétaldéhyde, de triméthylamine, de mercaptans, et de divers aldéhydes et acides.

Des actions simples peuvent être mises en œuvre pour limiter ces nuisances à la source : sensibilisation du personnel à l'hygiène, sélection des matières premières, réduction des temps de stockage, mise en dépression ou couverture de certains équipements (trémies, bacs), optimisation du fonctionnement des biofiltres...

Compte tenu des débits importants et de la nature des composés présents à des concentrations relativement faibles (comparativement à d'autres secteurs d'activités industrielles), le traitement privilégié pour les effluents gazeux issus de la production des corps gras animaux est la biofiltration. À ce jour, presque tous les sites sont équipés de systèmes élaborés (capotage des équipements, 
régulation hygrométrique). Le biofiltre est souvent précédé d'un traitement constitué d'un aérocondenseur et d'une tour de lavage.

On observe une amélioration de l'entretien des équipements actuels (biofiltres traditionnels) et des projets d'équipement en biofiltre avec support minéral sont en cours. Les nuisances olfactives générées par les effluents aqueux sont également prises en compte.

Notons qu'un projet d'arrêté relatif aux usines d'équarrissage soumises à autorisation sous la rubrique 2730 de la nomenclature des installations classées pour la protection de l'environnement (traitement des cadavres, des déchets ou des sous-produits d'origine animale) risque d'imposer des prescriptions précises relatives aux nuisances olfactives.

Pour répondre également à d'autres exigences que la réduction des nuisances olfactives (destruction de co-produits, production d'énergie), les industriels s'équipent actuellement d'oxydeurs de buées.

\section{La gestion des déchets liés au process et le traitement des effluents aqueux}

La gestion des déchets est une des préoccupations majeures du secteur des corps gras, notamment pour le raffinage et pour la lipochimie, du fait des tonnages en sous-produits que ces activités génèrent.

La nature des déchets est diverse : déchets non spécifiques, constitués de DIB (déchets industriels banals) en mélange, déchets d'emballage, big bags, ferrailles, solvants, mais surtout déchets de process, et plus particulièrement les déchets dits " graisseux ", sur lesquels nous porterons notre attention.

\section{La trituration des graines oléagineuses}

Dans le cas de la trituration des graines oléagineuses, les seuls déchets de process identifiés sont un mélange de tourteaux humides, pailles et poussières avec éventuellement des graines étrangères et d'autres produits divers. Ils trouvent leur origine au cours des différentes étapes de manutention des graines et tourteaux. Ces déchets sont valorisés en alimentation animale dans les tourteaux, qui ne doivent pas contenir plus de $1 \%$ de poussières, mais aussi en incinération. Le gisement annuel est de l'ordre de 850 tonnes [7]. Les sous-produits de la pression sont directement réintroduits dans le circuit de fabrication : pieds de presse au niveau des presses, résidus du tamisage au niveau du cuiseur.

\section{Le raffinage}

Le raffinage des huiles végétales génère des déchets à différentes étapes (figure 1). Le gisement national est présenté tableau 2. Dans le cas du raffinage physique, les mucilages séparés au niveau de la centrifugeuse sont réincorporés dans les tourteaux lorsque les unités de trituration et de raffinage se situent sur le même site. Les mucilages peuvent être séchés sous vide et purifiés afin d'obtenir des lécithines valorisables (entre 50 et $90 \%$ du prix de l'huile brute). Dans le cas du raffinage chimique, les mucilages font partie intégrante des pâtes de neutralisation et suivent ainsi la même filière de valorisation que ces dernières. 
Le tonnage de pâtes de neutralisation généré varie en fonction de la qualité des huiles brutes. Le traitement généralement suivi consiste en la production d'huile acide par hydrolyse sulfurique.

Les terres minérales activées sont utilisées pour le raffinage des corps gras lors de la décoloration et dans certains cas lors de la wintérisation (décirage des huiles de tournesol et de maïs). Après usage, elles sont composées essentiellement de matière minérale (entre 30 et $60 \%$ ), de matière grasse (environ 50 \%) et d'eau (jusqu'à $10 \%$ ). La matière grasse est altérée, ce qui confère à ces terres un caractère d'inflammabilité spontanée que l'industriel doit considérer lors du stockage (nécessité d'arroser les terres et de les conditionner, en big bag par exemple, pour limiter le contact avec l'oxygène de l'air).

Le gisement français est estimé à environ 10000 tonnes par an [8], mais les quantités générées par site sont très variables (de 20 à plus de 2000 t/an).

La méthanisation est la filière la plus répandue actuellement pour ce déchet, mais génère des coûts de transport très élevés, les unités de traitement étant situées au Danemark. Nous étudions actuellement les possibilités de développer une filière en France. Parallèlement à la valorisation énergétique, la faisabilité de valorisation matière est explorée, telle que l'utilisation de ces terres en tant que savon abrasif par exemple [9].

Les graisses d'effluents peuvent être valorisées de différentes manières :

- l'industriel dispose d'un atelier de cassage des pâtes de neutralisation ; il traite alors ses graisses d'effluents avec les sous-produits du raffinage pour obtenir des huiles acides ;

- l'atelier de raffinage est implanté sur le même site que l'atelier de trituration, permettant ainsi un recyclage dans les tourteaux ;

- les graisses d'effluents peuvent aussi faire l'objet d'un épandage.

La très grande majorité des graisses d'effluents est valorisée avec les soapstocks. L'incorporation dans les tourteaux et l'épandage (lors des arrêts techniques de la trituration) sont deux filières relativement marginales et concernent les graisses d'effluents constituées des eaux cireuses. Le recyclage dans les tourteaux, au niveau de la pelletisation, permet un apport en eau améliorant l'efficacité de cette étape.

Les volumes d'effluents aqueux générés sur les sites de raffinage sont très variables et dépendent de la mise en place du recyclage des eaux de refroidissement (de plus en plus pratiqué) et des eaux traitées, mais aussi du type de raffinage (physique ou chimique).

La totalité des installations de raffinage est équipée de procédés de traitement, plus ou moins performants, de leurs eaux polluées. Ces dernières subissent en règle générale un traitement physico-chimique, très occasionnellement complété par un traitement biologique, avant d'être rejetées dans une station communale ou dans le milieu naturel. Dans certains cas, le respect des contraintes réglementaires pose de réelles difficultés aux industriels (par exemple : élimination des boues issues d'un traitement biologique). 
Les principaux déchets résultant de ce secteur sont liés à l'activité éventuelle de raffinage (tableau 3). Le gisement d'eaux gélatineuses issues de l'épuration des effluents aqueux varie en fonction du type de traitement retenu par les industriels : le procédé de fonte humide, principalement utilisé, est fortement consommateur d'eau ; le procédé de fonte à sec permet un recyclage de l'eau utilisée comme fluide thermique.

Les eaux gélatineuses, représentant environ $35 \%$ de la matière première traitée, sont concentrées et peuvent être incorporées dans le process. Nous étudions actuellement les possibilités de leur valorisation en méthanisation. Les effluents aqueux subissent une épuration biologique ou un prétraitement physico-chimique avant raccordement à la station d'épuration communale, rejet en rivière ou épandage. Les boues générées sont le plus souvent incinérées, mais peuvent également être épandues.

Les graisses d'effluent récupérées au niveau des dégraisseurs statiques ou des aéroflottateurs sont principalement incinérées. Le gisement national peut être évalué à 10000 tonnes/an [10].

Ce secteur est confronté à d'incessantes mutations en ce qui concerne la gestion de ces rejets, de part l'évolution des contraintes réglementaires consécutives à la crise de I'ESB (encéphalopathie spongiforme bovine).

Le projet d'arrêté concernant l'activité de traitement de cadavres, des déchets ou des sous-produits d'origine animale impose pour les nouvelles installations le principe du " rejet zéro " pour tout effluent liquide autre que les eaux pluviales. Les installations existantes doivent être équipées d'un dispositif permettant de garantir l'absence de rejet liquide et l'innocuité des rejets gazeux ou alors respecter des normes de rejets fixées par l'arrêté et garantir l'innocuité de leurs rejets liquides, par la mise en place d'un dispositif scientifiquement validé et faisant l'objet d'une diffusion par instruction ministérielle.

Pour les installations dont la destruction des déchets est réglementairement obligatoire, les effluents liquides devront faire l'objet d'un autoclavage à $133^{\circ} \mathrm{C}$ pendant $20 \mathrm{~min}$ sous une pression de 3 bars et/ou d'un traitement par tout dispositif scientifiquement validé d'efficacité équivalente.

\section{La margarinerie}

Les seuls déchets de process rencontrés sont issus des loupés de fabrication après recyclage (500 tonnes par an) et les retours de date limite de consommation (tableau 4). Ils subissent une opération de refonte avant d'être valorisés. Les graisses d'effluents (1 700 tonnes/an) étaient encore valorisées en alimentation animale récemment.

L'eau est principalement utilisée pour la production de vapeur, le nettoyage des circuits des lignes de production et en fabrication (constitution de la phase aqueuse). L'effluent aqueux est essentiellement constitué des eaux de lavage. Les installations de traitement en place sont le plus souvent de simples décanteurs statiques, avant rejet dans une station communale. 


\section{La lipochimie}

La lipochimie peut générer des déchets spécifiques des matières premières et des process utilisés. La figure 2 permet de visualiser de manière synthétique l'origine de ces déchets.

Concernant les résidus de filtration, les quantités produites sont très variables suivant les procédés utilisés : de 0,1 à $20 \mathrm{~kg} / \mathrm{t}$ de produits finis [11]. Ils sont récupérés par des sociétés extérieures pour une mise en décharge, une valorisation matière ou énergétique (tableau 5). Cette catégorie de déchets comprend notamment les charbons actifs et les terres de décoloration usagées. Les coûts d'élimination varient considérablement en fonction du mode de traitement : l'incinération est le mode générant les coûts les plus importants.

La filière de traitement des résidus de distillation est l'incinération en externe. Un site cependant procède pour environ $50 \%$ de ces résidus de distillation à l'incinération en interne avec récupération de chaleur, le $\mathrm{PCl}$ (pouvoir calorifique inférieur) du produit étant d'environ $8000 \mathrm{kcal} / \mathrm{kg}$. Les coûts de traitement s'élèvent d'environ 230 à 410 euros par tonne de produit fini. Une entreprise se distingue en ayant négocié la reprise de ses résidus à coût nul.

Concernant les mélanges de résidus d'esters, d'eau et de glycérine (dans le cas de la production d'esters méthyliques) ou d'huiles acides, les principales filières sont l'incinération en cimenterie ou la valorisation matière en savonnerie (huiles acides). Les quantités produites sont très variables d'un site à l'autre : de 0,2 à $33 \mathrm{~kg} / \mathrm{t}$ de produit fini. La valorisation matière permet d'éliminer le produit sans frais. Les coûts de l'incinération en cimenterie sont très variables (de 107 à 305 euros/t de déchets).

Les eaux à traiter ont principalement pour origine le lavage (réacteurs et sol), le process, la production de vide, le refroidissement, les chaudières.

Les traitements mis en place vont de la simple décantation au traitement physico-chimique ou anaérobie. Les matières grasses résultant de la décantation sont parfois réincorporées dans le process. Les eaux traitées sont rejetées dans le milieu naturel ou dirigée vers une station communale de traitement.

Les volumes d'effluent à traiter sont très variables (de 8000 à $1300000 \mathrm{~m}^{3}$ ) et sont davantage liés au type de process mis en œuvre plutôt qu'à la quantité produite. Les débits vont de 3,8 à $53,3 \mathrm{~m}^{3}$ par tonne de produit fini. Des activités telles que le raffinage (nécessitant des lavages et la production de vide) sont très consommatrices d'eau.

Si les sites industriels les plus importants ont mis en place une politique de gestion satisfaisante, un accompagnement doit être proposé aux structures moins importantes qui ne disposent pas encore à I'heure actuelle des moyens, en termes de temps et de compétences, pour optimiser les filières. Un des points critique du secteur reste le traitement des eaux et impose la mise en place à court terme de systèmes assez lourds (station de traitement biologique par voie anaérobie suivi d'un épandage des boues). 


\section{L'énergie}

La trituration des graines oléagineuses

L'énergie est un élément stratégique pour la trituration des graines oléagineuses. L'étude réalisée par I'Iterg [12] fait apparaître que ce secteur a consommé au total 83500 tep (tonne équivalent pétrole) durant l'année 1995, pour 2820000 de tonnes de graines triturées. L'énergie électrique, sous forme d'énergie motrice, représente $30 \%$ de l'énergie totale facturée (soit 25000 tep). L'énergie thermique, utilisée en grande partie pour produire de la vapeur, représente $70 \%$ (soit 58500 tep) de l'énergie totale.

La vapeur est consommée massivement lors de la cuisson de la graine et en désolvantation des tourteaux. Le tableau 6 présente une estimation des répartitions d'énergie consommée par poste en fonction des puissances électriques installées et de l'usage de la vapeur.

Des efforts de recherche pour diminuer la consommation énergétique ont été réalisés ces dernières années : utilisation de presse de plus grande capacité, de broyeur ou aplatisseur plus résistants, de Désolvantisateur Toas-teur Dryer Cooler (DTDC) du type Schumacher plus performant, de prédésolvantiseur pour minimiser la consommation de vapeur.

La recherche d'une meilleure productivité est un élément essentiel qui dicte la stratégie des entreprises de ce secteur : regroupement, intégration de la trituration dans le raffinage et recherche d'économies de fonctionnement sont notamment des éléments clairement identifiés de cette stratégie. À ce titre, l'énergie est en particulier un poste important du coût de la transformation que le triturateur cherche à minimiser.

Le cas spécifique des petites huileries

Les petits huiliers français traitent, par pression simple, seulement $5 \%$ environ de la totalité des graines triturées en France, par conséquent la consommation d'énergie de ce secteur est relativement faible; elle est estimée à 500 tep.

Dans ce secteur, la part de l'énergie électrique consommée est légèrement supérieure (environ $45 \%$ de la consommation totale) à celle de l'énergie thermique. Cela s'explique par le fait qu'une majorité d'huiliers ne réalisent que de la pression sans cuisson préalable, d'où une demande quasi exclusive d'électricité pour le fonctionnement de l'atelier de pression. La répartition exacte de l'utilisation de l'énergie en fonction du poste est difficile à établir du fait de l'absence de compteurs divisionnaires et de données répertoriées.

Compte tenu de la faible part de l'énergie dans le coût de la transformation de la graine et de la marge dégagée par le produit vendu, la sensibilité de l'huilier vis-à-vis des économies d'énergie est moindre.

Le raffinage des huiles végétales

En 1995, la consommation de l'activité de raffinage s'élève à 25394 tep, pour une production de 577 000 tonnes d'huile [12]. L'énergie électrique sous forme d'énergie motrice représente $27 \%$ de l'énergie totale facturée (soit 6840 tep). L'énergie thermique, utilisée essentiellement pour produire de la vapeur, en représente $73 \%$ (soit 18554 tep). En règle générale, les consommations spécifiques 
diminuent en fonction de l'importance des installations, ce qui s'explique par les économies d'échelle réalisées par les installations de grande taille. II apparaît que la part de l'énergie électrique est plus importante dans les installations de forte capacité, les installations de plus faible capacité n'ayant pas optimisé leur utilisation de vapeur (gaz naturel pour les installations importantes, fuel lourd pour encore quelques petites unités).

Les répartitions entre les différentes énergies (électrique et thermique) ne varient pas suivant le type d'huile raffinée. En revanche, on observe une évolution de la consommation totale d'énergie suivant le type d'huile mis en œuvre. Il apparaît que le raffinage de l'huile de tournesol est moins consommateur que celui des huiles de colza ou d'arachide, ce qui peut être à priori considéré comme paradoxal, étant donné que le traitement de l'huile de tournesol intègre l'étape supplémentaire de décirage. Notons cependant que les huiles de colza et d'arachide nécessitent généralement un prétraitement plus poussé avec des temps de contact plus importants et une désodorisation à température plus élevée avec un temps de séjour plus long.

\section{La production des corps gras animaux}

Suite à une étude que nous avons menée sur des données de 1998 [7], il apparaît que la consommation totale d'énergie s'élève à 57000 tep par an, avec 79 \% pour l'énergie thermique (45 000 tep) et $21 \%$ pour l'énergie motrice (soit 12000 tep), pour une production de l'ordre de 500000 tonnes. Les consommations varient de manière très importantes d'un site à l'autre, en fonction de la capacité de production et du procédé de fabrication : la consommation totale des procédés de fonte humide est supérieure à celle des procédés de fonte à sec. L'énergie est un poste important pour ce secteur, mais ne constitue pas une préoccupation environnementale majeure.

\section{La lipochimie}

La production de ce secteur en 1999 est de l'ordre de 750000 tonnes de produits finis. La quantité produite est très variable selon les sites : elle s'étend de moins de 2000 tonnes à environ 200000 tonnes par an. Les plus petites unités de production ont généralement une large gamme de production. Les réactions de transformation dans ce secteur sont réalisées dans des conditions opératoires pouvant être très consommatrices d'énergie. Par exemple, les alcools gras sont produits à $250^{\circ} \mathrm{C}$ sous 300 bars en présence d'un catalyseur.

La consommation en énergie motrice d'un site est très variable puisqu'elle évolue d'environ 1800 à $23000 \mathrm{MWh}$. Elle n'est pas liée à la quantité de matière produite, puisque cette consommation varie de 48 à $1219 \mathrm{kWh} / \mathrm{t}$ (la moyenne étant de $110 \mathrm{kWh} / \mathrm{t}$ ). Le plus petit site de production reste cependant celui consommant le plus d'énergie motrice pour produire une tonne. Au niveau national, la consommation d'énergie motrice de ce secteur est estimée à 18375 tep [11].

Le gaz naturel est la principale source d'énergie thermique (98\% de l'ensemble des sources, en tep). Les consommations en énergie thermique sont très variables et restent indépendantes de la quantité produite : elles varient de 0,020 à 0,111 tep/t produite, avec une moyenne à 0,055 tep/tonne produite. Au niveau national, la consommation d'énergie thermique de ce secteur est estimée à 40 916 tep.

La production totale de ce secteur est de 59300 tep, avec $31 \%$ consacrés à l'énergie motrice et $69 \%$ consacrés à l'énergie thermique. Le coût de l'énergie représente 12,6 euros par tonne produite. Cette 
moyenne cache des écarts importants : la fourchette s'étend selon les sites de 4,6 euros à 83 euros/t. Les plus petits sites étant, proportionnellement, les plus gros consommateurs.

Les industriels estiment que leurs consommations en énergie ont évolué au cours des trois dernières années et sont justifiées par celles de la production, par des modifications du process, une diversification des activités, mais aussi parfois par des exigences en matière de qualité.

Afin de réduire ces consommations, les points à améliorer sont la récupération des condensats de vapeur, l'installation d'échangeurs supplémentaires au niveau des installations de distillation, et le passage au gaz naturel.

Les principales évolutions en termes de consommation d'énergie dans ce secteur sont, pour les années à venir, l'utilisation d'unités de cogénération pour les sites industriels d'une certaine importance. Les sites de moins grande importance doivent maintenant bénéficier d'actions de sensibilisation visant à induire une prise de conscience et une réflexion sur les consommations. Ces actions devront s'accompagner de la réalisation de diagnostics et de campagne de mesures et la pose systématique de compteurs divisionnaires.

\section{L'utilisation des fluides frigorigènes}

Cette problématique, bien que non spécifique à notre profession, intéresse néanmoins au premier chef les industriels de la margarinerie, ceux exerçant une activité de fractionnement de matière grasse, de stockage en chambre froide et plus globalement les utilisateurs de groupes de froid. La technique du froid industriel, telle qu'elle est pratiquée aujourd'hui, demeure tout entière soumise au développement des fluides frigorigènes qui en assurent la fonction fondamentale de transfert d'énergie. George Vrinat [13] dresse un historique très complet de leur évolution, en distinguant quatre grandes périodes : " la protohistoire » de 1755 à 1930, " la belle époque " de 1930 à 1987, " les années folles » de 1987 à nos jours et le retour aux sources aujourd'hui et demain.

Après le temps des découvertes des procédés de froid industriels, suit une vaste période (1930-1987) de développement de l'utilisation des CFC (chloro-fluoro carbonés) suite à la création des fréons par la société Dupont de Nemours.

En 1987, des actes réglementaires voient le jour à propos tout d'abord de la réduction de la couche d'ozone, au centre des préoccupations de la communauté internationale. L'activité de destruction de la couche d'ozone par un gaz est notamment caractérisée par son ODP (ozone depletion potential) qui est très élevé pour les CFC. II apparaît que ces derniers apportent un effet réverbérant sur la chaleur ré-émise la nuit par la terre de 100 à 1000 fois plus important que celui du $\mathrm{CO}_{2}$ produit par l'industrie. II en résulte la création des fluides de transition, les HCFC remplacés plus récemment par des produits de substitution plus pérennes les HFC ne renfermant plus de chlore.

La réglementation européenne prévoit l'éradication progressive des CFC et des HCFC selon le calendrier précis : pour les CFC, l'arrêt de la commercialisation doit être effective depuis le $1^{\text {er }}$ janvier 2000 et l'arrêt de l'utilisation en vue de la maintenance et de l'entretien doit l'être depuis le $1^{\text {er }}$ janvier 2001 ; pour les HCFC, l'arrêt de l'utilisation en vue de la maintenance et de l'entretien devra être effective au $1^{\mathrm{er}}$ janvier 2010. 
Cependant, même s'il n'est pas menacé à court terme, l'avenir des HFC reste incertain : si leur l'ODP est nul, leur GWP (global warming potential, paramètre intervenant dans l'évaluation de l'effet de serre) est élevé. Cela relance notamment l'intérêt de l'utilisation de l'ammoniac qui est considéré comme parmi les meilleurs fluides frigorigènes, et ce malgré une réglementation spécifique sévère liée à sa toxicité et son inflammabilité. Par ailleurs de nouveaux challenges sont à relever afin d'intégrer de nouveaux systèmes économisant l'énergie (indépendamment du fluide employé), une application rigoureuse des règles de construction du matériel mis en œuvre, et une qualification du personnel de maintenance pour garantir un confinement absolu des circuits, et enfin une gestion économique adaptée.

\section{CONCLUSION}

Nous pouvons hiérarchiser les préoccupations environnementales de l'industrie des corps gras de la manière suivante :

- la conformité réglementaire,

- la gestion des déchets industriels,

- le traitement des effluents aqueux,

- la maîtrise des effluents gazeux (émissions de COV et odeurs),

- la maîtrise de l'énergie,

- la mise en place d'un système de management environnemental.

Les contraintes réglementaires dans le domaine environnemental s'accentuent au fil des ans : la production annuelle de textes réglementaires relatifs à l'environnement (français et européens) était inférieure à 50 en 1971, voisine de 350 en 1993 et a dépassé 450 en 2000. La législation des installations classées s'articule avec le droit de l'urbanisme, la loi sur l'eau, la loi sur les déchets, les lois de finances (pour la taxe générale sur les activités polluante), le Code du travail, la réglementation concernant le transport... Cela oblige l'exploitant industriel, responsable pénalement, à vérifier quotidiennement la conformité réglementaire de son site et à réaliser régulièrement des modifications de ces installations.

La gestion des déchets reste une préoccupation essentielle, tout particulièrement pour le secteur des corps gras animaux et la lipochimie, en raison de la remise en cause des filières d'élimination due au renforcement des contraintes réglementaires, mais aussi en raison coûts de traitement. Ce poste ne doit pas être négligé par les entreprises : réduction à la source, recherche de filières pérennes, valorisation énergétique ou matière sont des éléments, certes consommateurs de temps, mais permettant une réduction significative des coûts. Notons que dans le secteur de la lipochime, un choix optimisé des filières de traitement peut permettre de réduire les coûts d'un facteur 3 .

Des avancées notables sont observées dans le domaine du traitement de l'eau depuis une décennie : des difficultés persistent, notamment dans les domaines des corps gras animaux et celui de la lipochimie, que des projets de création de stations d'épuration devraient résoudre. 
Les nuisances olfactives sont maintenant considérées par les exploitants, avec la mise en place de traitement complexes et efficaces, notamment dans le secteur de la production des corps gras animaux. Les autres secteurs ne doivent pas négliger les gênes subies par un environnement sensible et des actions simples peuvent parfois solutionner des relations conflictuelles avec le voisinage. Le diagnostic environnemental trouve ici sa place.

L'aspect énergétique, s'il a été pris en compte lors des grandes crises pétrolières, est aujourd'hui négligé. Une stratégie politique d'incitation économique doit pouvoir remédier à ce manque. L'Iterg entend développer prochainement des actions de sensibilisation dans ce domaine auprès des industriels.

La meilleure méthode pour prendre en compte l'ensemble des ces aspects environnementaux reste bien évidemment la mise en place d'un système de management environnemental. Si seulement quelques sites sont certifiés à ce jour, de nombreuses démarches sont en cours et les industriels prennent peu à peu conscience de l'intérêt d'une politique basée sur un référentiel. L'approche globale proposée par les systèmes de management intégré qualité/sécurité/environnement reste certainement la voie garantissant une gestion efficace et durable.

\section{Remerciements}

Le panorama des préoccupations environnementales de notre profession a pu être dressé à partir de différentes études réalisées par l'Iterg depuis 1994, avec l'appui financier de la profession et de divers organismes. Nous remercions tout particulièrement Didier Majou, de I'ACTIA (Association de coordination technique pour l'industrie agro-alimentaire), et Bernard Martelly, de la Direction de l'Agriculture et des Bioénergies de l'ADEME (Agence de l'environnement et de la maîtrise de l'énergie) pour leurs soutiens précieux et constructifs.

\section{REFERENCES}

1. JOHNSON LA (1997). Theorical, comparative, and historical analyses of alternative technologies for oilseeds extraction. In : Wan PJ, Wakelyn PJ, eds. Technology and solvents for extracting oilseeds and nonpetrolum oils. AOCS Press, 4-47.

2. VALLAUD A, SALMON P (1967). Sécurité et hygiène dans l'industrie de I'huilerie, éd. INRS, 37-47.

3. GALVIN JB (1967). Toxicity data for commercial hexane and hexane isomers. In : Wan PJ, Wakelyn PJ, eds. Technology and solvents for extracting oilseeds and nonpetrolum oils. AOCS Press, 75-85.

4. LUSAS EW, HERNANDEZ E (1967). Isopropyl alcohol. In : Wan PJ, Wakelyn PJ, eds. Technology and solvents for extracting oilseeds and nonpetrolum oils. AOCS Press, 199-266.

5. LACOSTE F, BOSQUE F, BRENNE E (1996). Contrôle des émissions atmosphériques d'unités industrielles de trituration de colza. OCL, $3: 369-77$.

6. ITERG (2001). Caractérisation des odeurs émises par l'agro-industrie et détermination des traitements appropriés. Travaux non publiés.

7. ITERG (1999). Contrat de secteur ADEME/Iterg Énergie - Environnement, programme 1998-1999. Travaux non publiés. 
8. ITERG (1999). Les terres usagées issues du raffinage des corps gras - situation actuelle en France et perspectives. Travaux non publiés.

9. PAGES X, GUILLON O (2001). Composition récurrente du type savon abrasif et procédé de production d'une telle composition. Demande de brevet international n PCT/FRO1/01224.

10. ITERG (1998). Gisement et valorisation des déchets graisseux dans le secteur des corps gras. Travaux non publiés.

11. ITERG (2000). Valorisation énergétique et matière des déchets dans le secteur de la lipochimie. Travaux non publiés.

12. ITERG (1997). Contrat de secteur ADEME/Iterg, programme 1996-1997. Travaux non publiés.

13. VRINAT G (2001). Les fluides à faible effet de serre. Revue Générale du Froid, 1010 : 29-34.

Illustrations

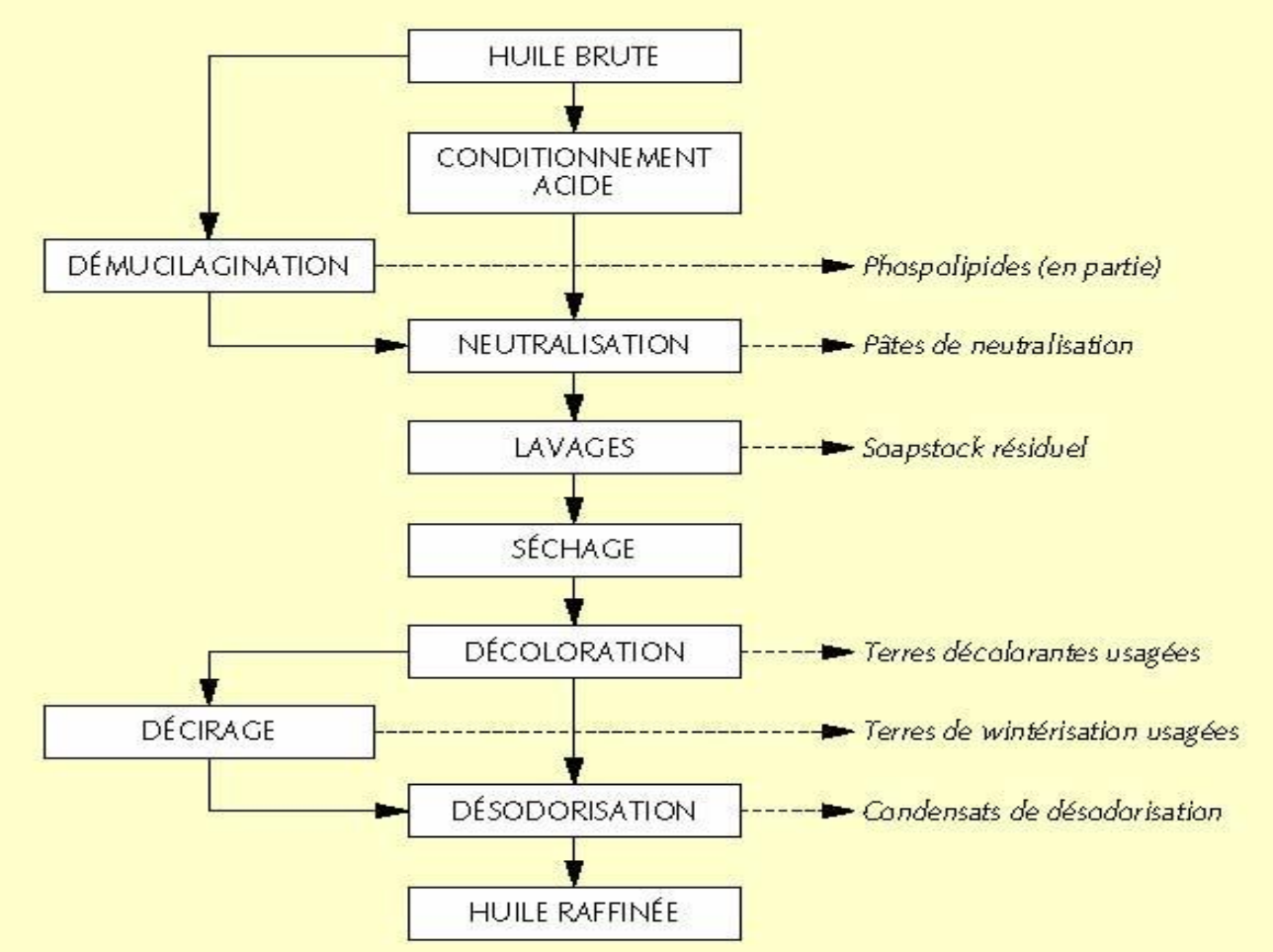

Figure 1. Sources des sous-produits issus du raffinage chimique. 


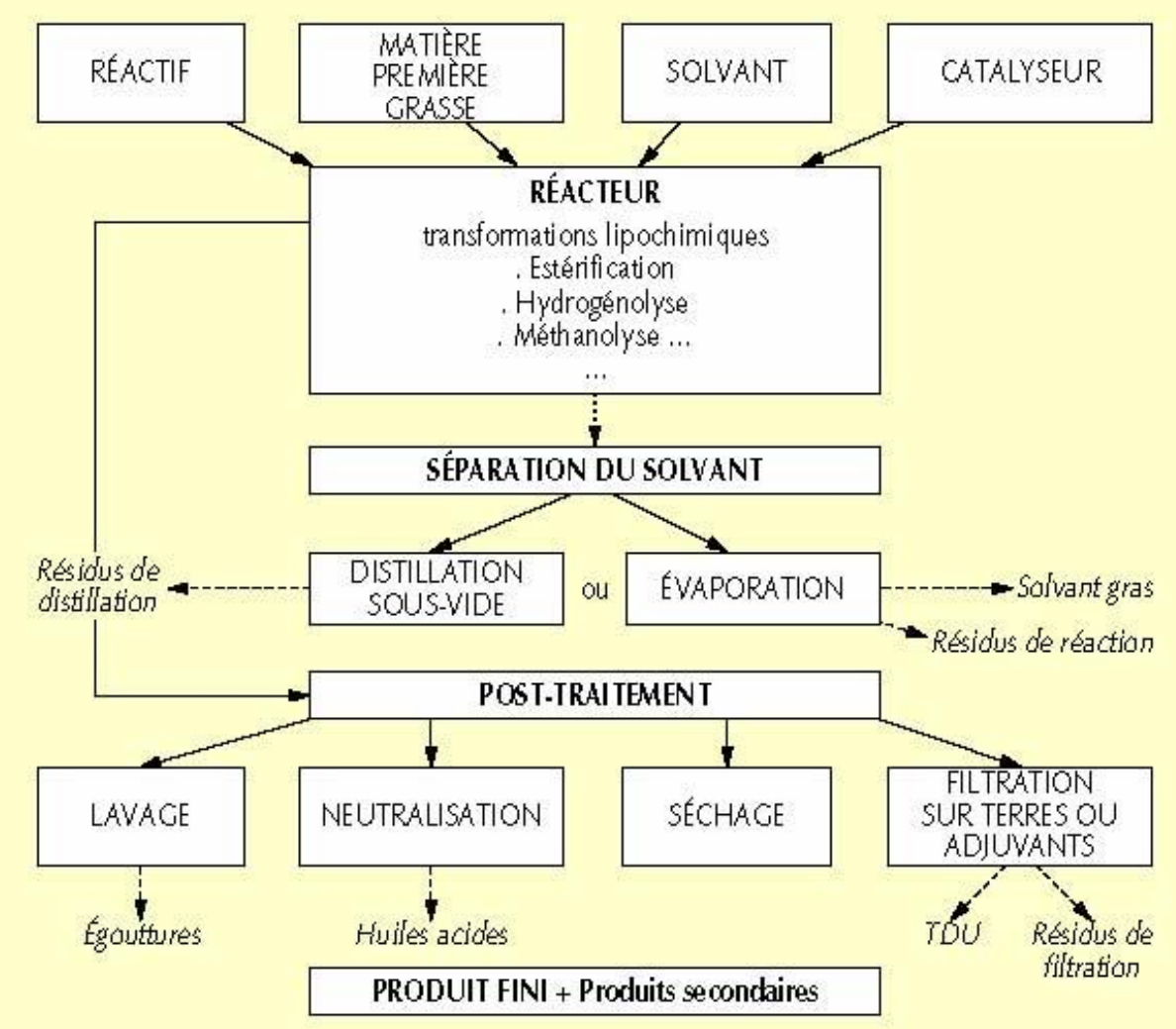

Figure 2. Origine des déchets liés aux process utilisés en lipochimie.

Tableau 1. Valeur limite d'émission des émissions de COV (cos des consommations de solvants supérieures à 10 to nnes par an).

\begin{tabular}{|c|c|}
\hline Type de matière traitée & $\begin{array}{c}\text { Valeur limite d'émission totale de COV } \\
\text { par tonn e de matière traitée }\end{array}$ \\
\hline Graisse animale & $1,5 \mathrm{~kg} /$ tonne \\
\hline Ricin & $3 \mathrm{~kg} /$ tonne \\
\hline Colza & $1 \mathrm{~kg} /$ tonne \\
\hline Toumesol & $1 \mathrm{~kg} /$ tonne \\
\hline $\begin{array}{l}\text { Soja } \\
\text { (broyage normal) }\end{array}$ & $0,8 \mathrm{~kg} /$ tonne \\
\hline $\begin{array}{l}\text { Soja } \\
\text { (floconsblancs) }\end{array}$ & $1,2 \mathrm{~kg} /$ tonne \\
\hline $\begin{array}{l}\text { Autres graines } \\
\text { et autres matières végéta les }\end{array}$ & $3 \mathrm{~kg} /$ tonne $1,2,3$ \\
\hline
\end{tabular}

1 Pour les irstal bations trarsformant des bts sépa rés, les valeurs limites d’émission sont fixée: au cas par cas en recourant aux meilleures techniques dis ponibles.

2 Pour les procédés de fractionnement, à l'exception de la démucibagination (élimination de: mat ières qomme uses de l' huile), le total des émissions est inférieur ou éqal à $1,5 \mathrm{~kg} /$ tonne. 'Pour b démucilag ination, lo totaldes émissions est inférieur ou égal à $4 \mathrm{~kg} /$ tonne. 
Tableau 2. Gisement national des principaux déchets issus dur roffinage des huiles végétales [7].

\begin{tabular}{|l|c|l}
\hline \multicolumn{1}{|c|}{ Type de déch ets } & $\begin{array}{c}\text { Gisem ent national } \\
\text { tonn e/ an }\end{array}$ & \multicolumn{1}{|c}{ Filière de valorisation } \\
\hline Gommes ou mucilages & 4000 & $\begin{array}{l}\text { Production de lécithine - recyclage } \\
\text { dans les tourteaux }\end{array}$ \\
Soapstocks & 19000 & $\begin{array}{l}\text { Production d'huiles acides } \\
\text { (savonnerie, lipochimie...) } \\
\text { Méthanisation - compostage - } \\
\text { centre de stoclage... }\end{array}$ \\
Terres décolorantes uggées & 2800 & $\begin{array}{l}\text { Vabrisés avec les huiles acides } \\
\text { Valorisation en savonnerie, lipochimie... }\end{array}$ \\
$\begin{array}{l}\text { Terres de wintérisation usagées } \\
\text { Condensatsde désodorigation } \\
\text { Acides gras issus du raffinage } \\
\text { physiq ue } \\
\text { Graisses d'effluents }\end{array}$ & 17000 & $\begin{array}{l}\text { Recyclage da ns l'atelier de traitement } \\
\text { des soapstocks... }\end{array}$ \\
\hline
\end{tabular}

Tableau 3. Gisement notional des principarx déchets issus de la production de corps gas animoux [7].

\begin{tabular}{|c|c|c|}
\hline Type de déchets & Gisem ent national & $\begin{array}{l}\text { Filirère de traitem ent } \\
\text { tonn } \mathrm{e} / \text { an }\end{array}$ \\
\hline DIB en mélange & 225 & Centre de stockage \\
\hline Terres décolorantes uggées & 1000 & Méthanisation, centre de stockage \\
\hline Soapstocks & 3900 & Production d'huiles acides \\
\hline Condensatsde désodorigation & 70 & Vabrisation matière \\
\hline Graisses d'effluents & 10000 & Incinération \\
\hline Eaux gélatineuses & * & $\begin{array}{l}\text { Recyclage da ns le process épandage - } \\
\text { incinération }\end{array}$ \\
\hline Boues d'épuration & * & Incinération - épandage \\
\hline
\end{tabular}

- En cours d'évaluation.

Tableau 4. Gisement national des principara déchets de la margarinerie [7].

\begin{tabular}{|c|c|c|}
\hline Type de déchets & Gisement national & $\begin{array}{l}\text { Filière de traitem ent } \\
\text { tonn } \mathrm{e} / \text { an }\end{array}$ \\
\hline $\begin{array}{l}\text { Déchets de refonte des bupés } \\
\text { de fabrication }\end{array}$ & 500 & $\begin{array}{l}\text { Incinération, compostage, } \\
\text { centre de stoclage }\end{array}$ \\
\hline DIB en mélange & 105 & Centre de stockage, incinération \\
\hline Gartons de livaison des emballages & 650 & Réemploi externe \\
\hline Pa lettes & 290 & $\begin{array}{l}\text { Réemploi, rexyclage matériaux, } \\
\text { valorisation énergétique }\end{array}$ \\
\hline Big bags & 800 & Réemploi externe \\
\hline Solvants & 8 & Vabrisation énergétique \\
\hline Huiles minérales & 6 & Recyclage \\
\hline Ferraille & 140 & Recyclage \\
\hline Graisses d'effluents & 1700 & Alimentation animale \\
\hline
\end{tabular}


Tableau 5. Gisement notional des principarx déchets issus de la lipochömie [1 1 ].

\begin{tabular}{|c|c|c|c|c|}
\hline Nature & $\begin{array}{c}\text { Ratio } \\
\text { (kg/t de produits fin is) }\end{array}$ & $\begin{array}{c}\text { Gisem ent national } \\
\text { (tonne/an) }\end{array}$ & Traitem ent & $\begin{array}{c}\text { Coût moyen } \\
(€ / t)\end{array}$ \\
\hline Huiles minérales ugagées & 0,050 & 35 & $\begin{array}{l}\text { Incinération } \\
\text { valorisation }\end{array}$ & - \\
\hline Déchets de laboratoires & 0,016 & 12 & Incinération & - \\
\hline DIB en mélange & 0,9 & 708 & $\begin{array}{l}\text { Centre } \\
\text { de stockage } \\
\text { incinération }\end{array}$ & 168 \\
\hline Emballages souilkés & 1,9 & 1500 & Incinération & 708 \\
\hline Résidus de filtration & 2,7 & 2000 & $\begin{array}{l}\text { Centre } \\
\text { de stockage } \\
\text { incinération } \\
\text { valorisation }\end{array}$ & 198 \\
\hline Résidus de distillation & 24,7 & 7500 & Incinération & 381 \\
\hline Solvants usagés de process & 0,16 & 120 & Incinération & - \\
\hline Autres déchets de process & 4,5 & 3300 & $\begin{array}{l}\text { Incinération } \\
\text { valorisation }\end{array}$ & 183 \\
\hline Graisses d'effluent & 2,3 & 1750 & Vabrisation & - \\
\hline Boues de step & 16,6 & 4500 & $\begin{array}{l}\text { Centre } \\
\text { de stockage } \\
\text { épandage }\end{array}$ & 115 \\
\hline Effluent aqueux & $9,4 \mathrm{~m}^{3}$ & $7050000 \mathrm{~m}^{3}$ & $\begin{array}{l}\text { Réseau ubain } \\
\text { milieu naturel }\end{array}$ & - \\
\hline
\end{tabular}

Tableau 6. Répartition de l'énergie par poste dars le secteur de la trituration[12].

\begin{tabular}{|l|c|c}
\hline \multicolumn{1}{|c|}{ Poste } & Energie électrique (\%) & Energie thermique (\%) \\
\hline Décorticage & 3,3 & - \\
Broyage & 3,3 & - \\
Aplatissage & 9,8 & - \\
Traitement thermique & 11,5 & 45 \\
Pressage & 36,0 & - \\
Pelletisation & 16,3 & 3 \\
Extraction/toa stage & 15,5 & 52 \\
Refroidissement tourteaux & 4,1 & - \\
Eclairage & 0,2 & - \\
\hline
\end{tabular}

